\title{
Annealing Studies of Nanoporous Si Thin Films Fabricated by Dry Etch
}

\author{
Qing Hao*, Yue Xiao and Fabian Javier Medina
}

In this work, high-temperature annealing above $1073 \mathrm{~K}$ has been carried out to study the possible shape change of nanoporous Si thin films. Under $1273 \mathrm{~K}$, it is found that the pore size can still be largely maintained when the nanoporous $\mathrm{Si}$ thin film is on a $\mathrm{SiO} / \mathrm{Si}_{2}$ substrate. However, the pore size can significantly shrink when the film is suspended. The contrast suggests that the $\mathrm{SiO}_{2} / \mathrm{Si}$ substrate can play an important role in maintaining the nanoporous patterns at a high temperature. This finding can be important for the high-temperature applications of these porous thin films, such as thermoelectric power generation.

Keywords: Keywords: Nanoporous material; High temperature; Deep reactive ion etching

Received 8 December 2019, Accepted 19 December 2019

DOI: $10.30919 / \mathrm{esmm} 5 \mathrm{f} 608$

In recent years, nanoporous Si films have been widely studied for their potential applications in thermoelectric energy conversion, heat guide and phonon focusing. For thermoelectric power generation and refrigeration, a low lattice thermal conductivity $k_{L}$ and thus thermal conductivity $k$ can be achieved mostly with pore-edge phonon scattering, as the classical phonon size effects. ${ }^{1,2}$ This leads to a high thermoelectric figure of merit, defined as $Z T=S^{2} \sigma T / k=S^{2} \sigma T /\left(k_{E}+k_{L}\right)$, where $S, \sigma, k_{E}$, and $T$ represent Seebeck coefficient, electrical conductivity, electronic thermal conductivity, and absolute temperature, respectively. ${ }^{3}$ In theoretical modeling, the optimized $Z T$ of an indirectbandgap material like $\mathrm{Si}$ usually occurs when the bandgap $E_{g}$ is 6-10 $k_{B} T$, with $k_{B}$ as the Boltzmann's constant., ${ }^{4}$ For Si with a large bandgap $E_{g} \approx 1.1 \mathrm{eV}$, the $Z T$ value would not saturate up to $1200 \mathrm{~K}$, beyond which the materials become less stable. The monotonously increased $Z T$ from 300 to $1200 \mathrm{~K}$ can be found in existing studies on nanostructured bulk Si. ${ }^{6,7}$ For general nanostructured Si, one common question here is whether these materials can be stable at a very high temperature for long-term operations. In numerous studies, it has been suggested that the pores may be largely closed or opened under hightemperature annealing, ${ }^{8,9}$ which indicates limited applications of these materials for power generation involving high temperatures.

In this work, the possible shape change of nanoporous $\mathrm{Si}$ thin films is systematically studied for different annealing conditions. As a $20-\mu \mathrm{m}$-long bridge with nanopores, the studied $\mathrm{Si}$ thin films are patterned by electron beam lithography and then drilled with deep reactive ion etching (DRIE). It is found that the existence of a $\mathrm{SiO}_{2} / \mathrm{Si}$ substrate can effectively keep the pore size, whereas notable nanopore contraction can be found right after the bridge suspension. More careful examination suggests the suppression of pore size change can be attributed to the nonwetting interface between a $\mathrm{Si}$ droplet and a $\mathrm{SiO}_{2}$ substrate, ${ }^{10}$ which effectively prevent the "flow" of soften $\mathrm{Si}$ on the

Department of Aerospace and Mechanical Engineering, University of Arizona, Tucson, AZ 85721, USA

*E-mail: qinghao@email.arizona.edu substrate.

Instead of using irregular nanoporous bulk $\mathrm{Si}$ etched electrochemically, ${ }^{8} 9$ the studied periodic nanoporous $\mathrm{Si}$ films were fabricated from the 220-nm-thick device layer of a silicon-on-insulator (SOI) wafer. The buried oxide layer was more than $2 \mu \mathrm{m}$ in its thickness. The initial pore diameter $d$ is fixed at $109.1 \pm 7 \mathrm{~nm}$, with a pitch of $201.9 \pm 3.7 \mathrm{~nm}$. These values consider the surface roughness of DRIE-drilled pores, where the minimum diameter $d_{\min }$ and maximum diameter $d_{\max }$ are averaged for each pore (see Fig. 3 also) and then statistically counted over many pores. Table 1 summarizes important sample information, including the pore diameter $d$ after annealing and further suspension, and the annealing condition. All annealing was carried out under $\mathrm{N}_{2}$ protection, with a gas flow rate of $90 \mathrm{scfm}$. Some samples were broken during the suspension process so that the pore diameters after suspension were not available. Annealed at $1273 \mathrm{~K}$ for 180 minutes, one nanoporous thin film was completely broken into pieces after suspension (Fig. 1) so that the pore diameters after the suspension were not listed. Similar problems were found for several samples in Table 1. Such fractures were attributed to the mismatch between the thermal expansion coefficients of the $\mathrm{Si}$ film and the $\mathrm{SiO}_{2} / \mathrm{Si}$ substrate. Si has a linear thermal expansion coefficient of $2.69 \times 10^{-6}-4.43 \times 10^{-6} / \mathrm{K}$ from $336 \mathrm{~K}$ to $1236.9 \mathrm{~K},{ }^{11}$ whereas $\mathrm{SiO}_{2}$ has a much lower value of $0.55 \times 10^{-6} / \mathrm{K}$ at room temperature. ${ }^{12}$

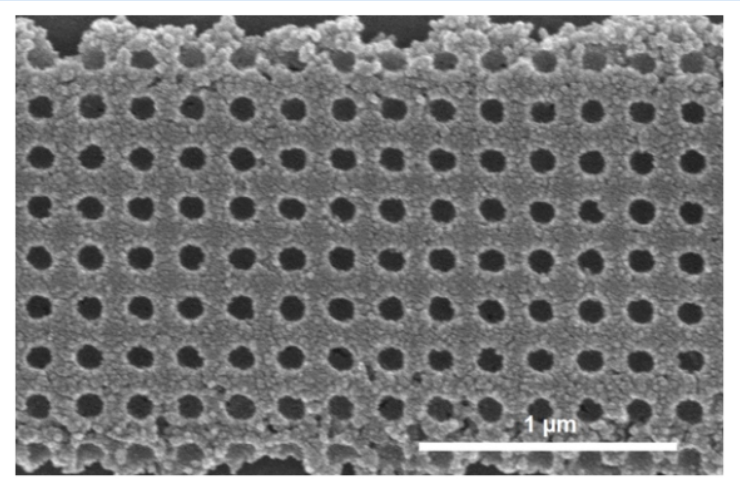

Fig. 1 The broken film after annealing at $1273 \mathrm{~K}$ for 180 mins. 

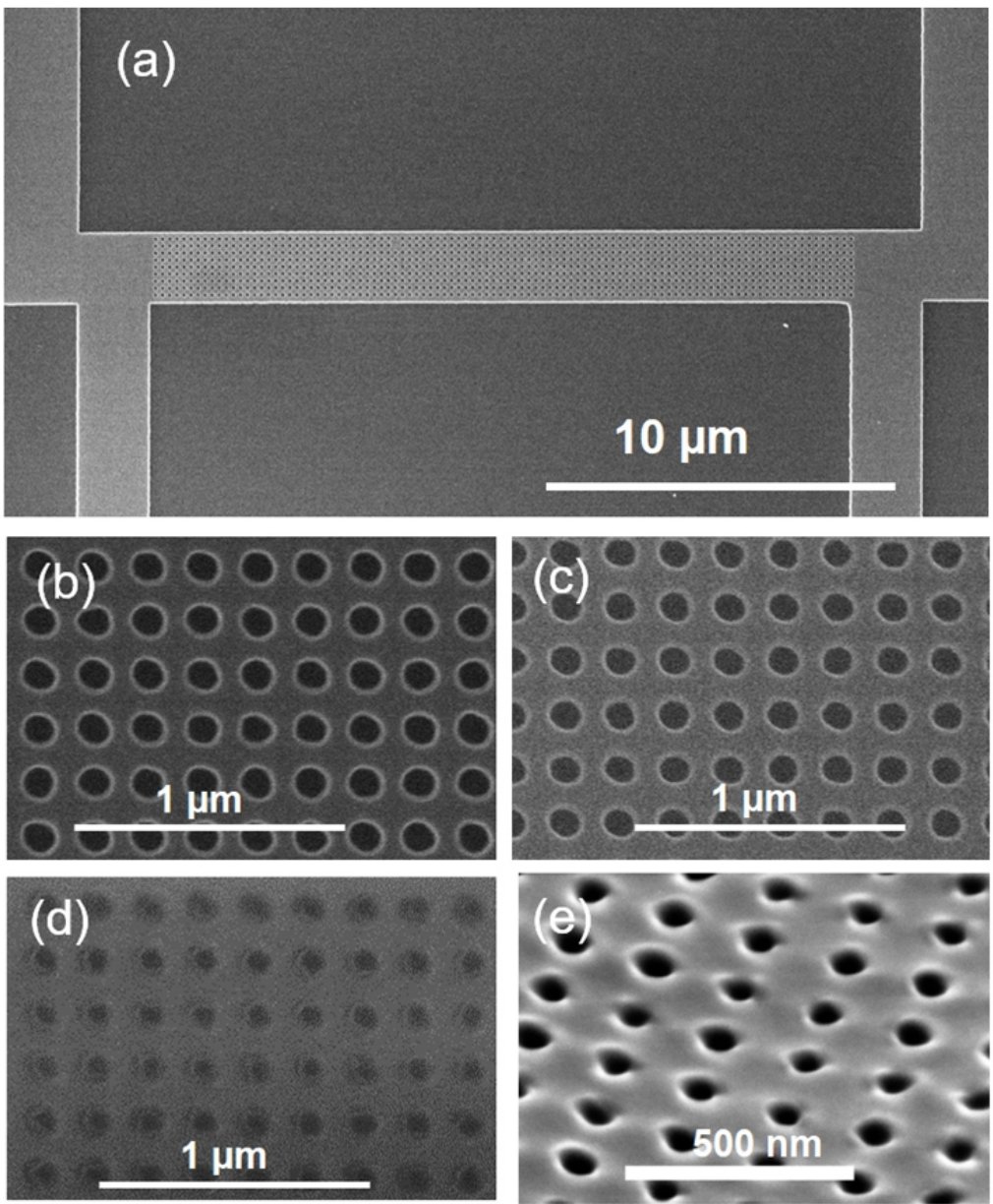

Fig. 2 (a) Top view of annealed but unsuspended Sample 2. Top-view SEM images of DRIE-drilled nanopores through the annealing and suspension process: (b) before annealing, (c) after annealing, and (d) after further suspension. (e) Tilted view of nanopores.

Table 1 Information about all studied samples. After suspension, Samples 2 and 4 have cracks in the middle but Sample 5 is well protected. Other samples are largely broken (e.g., Fig. 1) during the suspension so that the pore size is not reported.

\begin{tabular}{|c|c|c|c|}
\hline \multirow{2}{*}{ Sample \# } & \multicolumn{2}{|c|}{ Average Pore diameter $d_{a v g}(\mathrm{~nm})$} & \multirow[t]{2}{*}{ Annealing condition } \\
\hline & After annealing & After suspension & \\
\hline 1 & $118.8 \pm 8.3$ & N/A & $1073 \mathrm{~K}, 30$ minutes \\
\hline 2 & $118.3 \pm 7.3$ & $96.7 \pm 8.2$ & $1173 \mathrm{~K}, 30$ minutes \\
\hline 3 & $120.2 \pm 6.7$ & N/A & $1173 \mathrm{~K}, 60$ minutes \\
\hline 4 & $124.7 \pm 7.7$ & $93.7 \pm 8.3$ & $1223 \mathrm{~K}, 30$ minutes \\
\hline 5 & $124.0 \pm 7.4$ & $\begin{array}{c}47.5 \pm 15.1 \text { (middle region) to } \\
96.1 \pm 16.4 \text { (most region) }\end{array}$ & $1273 \mathrm{~K}, 30$ minutes \\
\hline 6 & $107.0 \pm 8.8$ & N/A & $1273 \mathrm{~K}, 120$ minutes \\
\hline 7 & $35.8 \pm 6.8$ & N/A & $1373 \mathrm{~K}, 30$ minutes \\
\hline
\end{tabular}


Fig. 2a shows one scanning electron microscopy (SEM) image for a typical thin-film device after suspension, as Sample 2 in Table 1. For electrochemical etching $\mathrm{Si}$ with random nanopores, experimental studies indicate pore expansion even under $673 \mathrm{~K}$ annealing for 45 minutes. ${ }^{8}$ For suspended nanoporous $\mathrm{SiO}_{2}$ films, annealing studies suggest a critical pore diameter $d_{C}$ as the film thickness. Below this $d_{C}$ value, pores would shrink under annealing, whereas pores with $d>d_{C}$ would expand during the annealing. ${ }^{13}, 14$ Different from these free-standing films, a Si film on $\mathrm{SiO}_{2}$ substrate may not have shape change due to the substrate influence. Because of the residual strain/stress within the annealed nanoporous structure, the nanopores shrunk right after the suspension even though no further annealing was carried out. For the studied 220nm-thick Si thin films, the bridge was easily broken in such suspension processes. In contrast, similar suspension was found to be usually safe for unannealed samples. ${ }^{15}$ Figs. 2b-d shows the detailed SEM images of Sample 2. These images were taken at three steps for the same region: 1) before annealing, 2) after annealing, and 3) after the bridge suspension. After annealing, the pore diameter expanded possibly due to the evaporation of the passive Teflon-like layer coated during the Bosch process of DRIE. ${ }^{16}$ After the suspension, the pore size was reduced from $118.3 \pm 7.3$ to $96.7 \pm 8.2 \mathrm{~nm}$. It should also be noted that the pore contraction may not be uniform after suspension. For Sample 5, nanopores in the middle had the largest contraction, whereas nanopores close to both ends of the bridge were less affected. For the other two samples annealed at a lower temperature, they were broken in the middle during the suspension and uniform pore contraction was found. Along with the nanopore contraction, a more complex surface undulation around each pore was observed after the suspension (Fig. 2d) though such features were not found before the suspension (Fig. 2c). A closer observation revealed some deformation of the thin film, as shown in Fig. 2e. Such deformation was associated with the strong contraction of the nanopores after the suspension.

The shape of the nanopore sidewall was also investigated. In the fabrication of a through-film nanopore on a 220-nm-thick Si thin film, two cycles of DRIE etching were performed. As the result, a wavy nanopore sidewall is expected, with a maximum diameter $d_{\max }$ and minimum diameter $d_{\text {min }}$. Fig. 3a shows the typical sidewall of a nanopore with a diameter of $\sim 200 \mathrm{~nm}$. However, softening of $\mathrm{Si}$ and the evaporation of the passive polymer layer during the high-temperature annealing lead to the shape change of the nanopore sidewall. Fig. $3 b$ reveals more information with the sidewall of Sample 5 after the annealing and suspension. The sidewall roughness is largely reduced after annealing and a more uniform pore diameter can be observed across the film thickness. In addition, the passivation-layer residuals during the Bosch process of DRIE (e.g., $\mathrm{C}_{4} \mathrm{~F}_{8}$ with a critical temperature of $388 \mathrm{~K}^{17}$ ) will be evaporated or thermally decomposed after the hightemperature annealing.

Despite the variation between different samples, it is clear that the $\mathrm{SiO}_{2} / \mathrm{Si}$ substrate can largely suppress the pore contraction during the annealing process and provide mechanical protection of the fragile nanoporous $\mathrm{Si}$ film under a high strain/stress. This effect can be understood from the large contact angle of $85^{\circ}$ between molten $\mathrm{Si}$ and a $\mathrm{SiO}_{2}$ substrate, ${ }^{18}$ indicating poor wetting for soften $\mathrm{Si}$ on $\mathrm{SiO}_{2}$. For ultrathin films, the strain field due to the $\mathrm{SiO}_{2}$ substrate may easily penetrate the film thickness so that a large strain field is anticipated across the whole nanoporous structure. For high-temperature applications, the impact of a high strain on the transport and other properties must be addressed.

In summary, nanoporous $\mathrm{Si}$ thin films on a $\mathrm{SiO}_{2} / \mathrm{Si}$ substrate were studies for the annealing impact on the pore sizes. It is found that the existence of the $\mathrm{SiO}_{2}$ substrate can effectively protect the nanofeatures during high-temperature annealing and significant pore contraction can be found right after the film suspension. In practice, such nanoporous thin films have been used for device cooling applications to effectively spread out the heat. ${ }^{19}$ For power generation, high temperatures are often involved and the nanofeatures can be preserved when these films are placed on $\mathrm{a} \mathrm{SiO}_{2}$ substrate. Such thermally insulated substrate can provide mechanical protection of a fragile nanoporous $\mathrm{Si}$ thin film, while minimizing the effective $Z T$ reduction due to additional thermal transport through the substrate. More fundamentally, compressive or tensive thermal strains are anticipated within heated thin films. More studies should be carried out on whether such strains can be used to benefit the thermoelectric applications. ${ }^{20,21}$

\section{Acknowledgements}

FIB characterizations, TEM and SEM analyses were performed at the Kuiper Materials Imaging and Characterization facility and the authors gratefully acknowledge NASA (grant numbers NNX12AL47G and
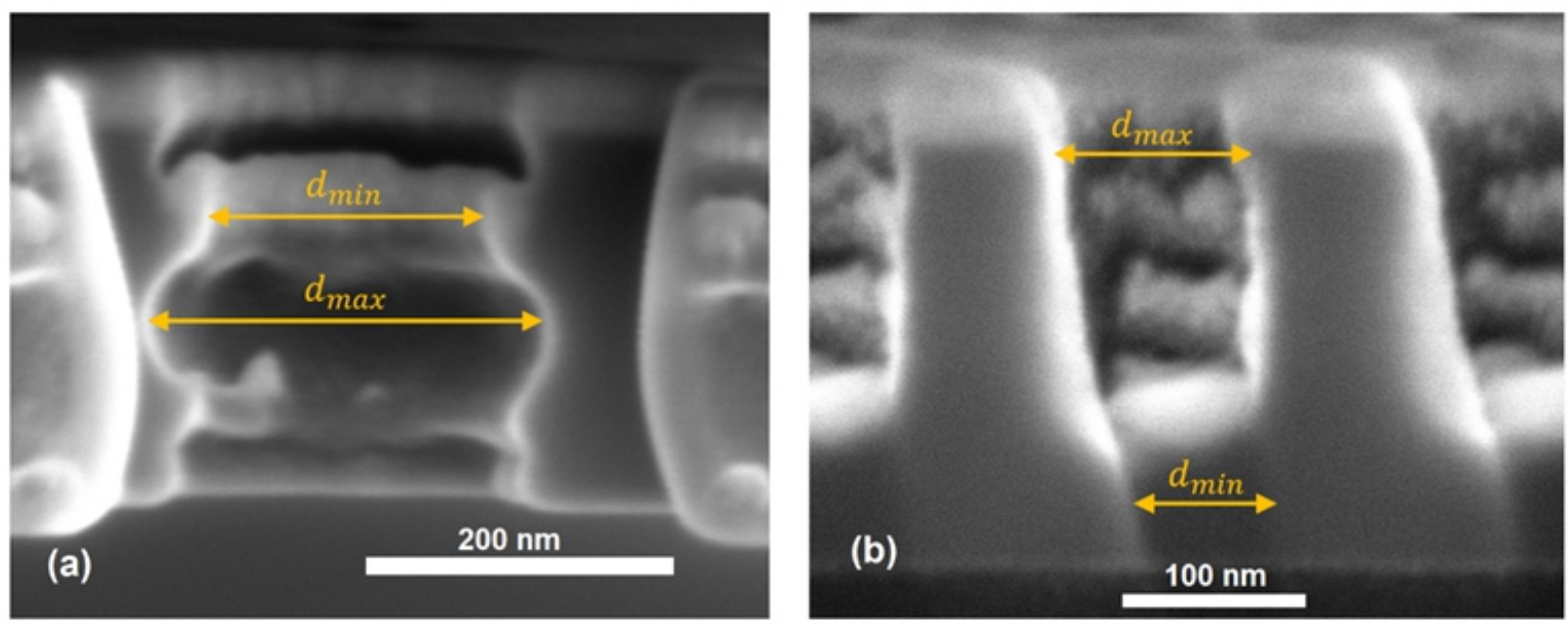

Fig. 3 (a) Cross-sectional SEM image of an unannealed 200-nm-diameter nanopore drilled by DRIE. (b) Cross-sectional SEM image of Sample 5 with annealing and suspension. Metal layers were coated onto both samples to discharge the sample during SEM studies. Key dimensions $d_{\text {min }}$ and $d_{\text {max }}$ are marked. 
NNX15AJ22G) and NSF (grant number 1531243) for funding of the instrumentation in the Kuiper Materials Imaging and Characterization Facility at the University of Arizona. This work has been supported by the U.S. Air Force Office of Scientific Research (grant number FA955016-1-0025) for studies of nanoporous structures, and the National Science Foundation (grant number CBET-1803931) for strain studies on nanostructures.

\section{References}

1. A. M. Marconnet, M. Asheghi and K. E. Goodson, J. Heat Tran., 2013, 135(6), 061601-061601/061610.

2. Y. Xiao, Q. Chen, D. Ma, N. Yang and Q. Hao, ES Mater.Manuf., 2019, 5, 2-18.

3. H. J. Goldsmid, Thermoelectric Refrigeration. (Plenum, New York, 1964).

4. R. Chasmar and R. Stratton,1959, Int. J. Electron., 7(1), 52-72.

5. G. D. Mahan, J. Appl. Phy., 1989, 65(4), 1578-1583.

6. S. K. Bux, R. G. Blair, P. K. Gogna, H. Lee, G. Chen, M. S. Dresselhaus, R. B. Kaner and J. P. Fleurial, Adv. Funct. Mater, 2009, 19(15), 2445-2452.

7. Q. Hao, D. Xu, N. Lu and H. Zhao, Phys. Rev. B, 2016, 93(20), 205206.

8. J. Salonen, E. Mäkilä, J. Riikonen, T. Heikkilä and V. P. Lehto, Phys. StatusSolidi A, 2009, 206(6), 1313-1317.
9. N. Ott, M. Nerding, G. Müller, R. Brendel and H. Strunk, J. Appl. Phys., 2004, 95(2), 497-503.

10. Z. Yuan, W. Huang and K. Mukai, Appl. Phys. A , 2004, 78(4), 617-622.

11. Y. Okada and Y. Tokumaru, J. Appl. Phys., 1984, 56(2), 314-320.

12. I. D. Sharp, D. O. Yi, Q. Xu, C. Y. Liao, J. W. Beeman, Z. Liliental-Weber, K. M. Yu, D. N. Zakharov, J. W. A. III, D. C. Chrzan and E. E. Haller, Appl. Phys. Lett., 2005, 86 (6), 063107.

13. A. Storm, J. Chen, X. Ling, H. Zandbergen and C. Dekker, Nat. Mater., 2003, 2(8), 537.

14. W. Asghar, A. Ilyas, J. A. Billo and S. M. Iqbal, Nanoscale Res. Lett., 2011, 6(1), 372.

15. Q. Hao, D. Xu, H. Zhao, Y. Xiao and F. J. Medina, Sci. Rep. 2018, 8(1), 9056.

16. B. Wu, A. Kumar and S. Pamarthy, J. Appl. Phys., 2010, 108(5), 9.

17. I. Praxair, edited by I. Praxair (2016).

18. Z. Yuan, W. L. Huang and K. Mukai, Appl. Phys. A, 2004, 78(4), 617-622.

19. Z. Ren, Z. Yu, J. C. Kim and J. Lee, Nanotechnology, 2018, 30(3), 035201.

20. S. V. Ovsyannikov and V. V. Shchennikov, Chem. Mater., 2009, 22(3), $635-$ 647.

21. B. Piccione and D. S. Gianola, Appl. Phys. Lett., 2015, 106 (11), 113101.

Publisher's Note Engineered Science Publisher remains neutral with regard to jurisdictional claims in published maps and institutional affiliations. 